\title{
Preface
}

\section{Tell me something I don't know}

When, in conversation, we cover something already familiar to the other person, we may hear the somewhat derogatory comment, "Tell me something I don't know."

In this issue on "Marfan Syndrome", we have enlisted the absolute world's authorities to expound on this disease. We are confident that every reader, regardless of level of sophistication, will find material not previously known to him or her. That was certainly true for your Editors, who learned a great deal in reviewing the chapters by these expert authors.

What a thrill it was to read the first-hand account by Reed Pyeritz of the discovery of the genetic and pathophysiologic mechanisms of Marfan disease, in which Dr. Pyeritz personally participated under the guidance of the revered Dr. Victor McKusick, the father of modern clinical genetics.

From Dr. Loeys himself and his team, we learn the fine nuances that can be employed to differentiate between the three easily confounded diseases: Marfan syndrome, Loeys-Dietz syndrome, and Ehlers-Danlos syndrome.

Since fibrillin is important to structural integrity throughout the body, Marfan disease can have implications for many non-cardiac organ systems. Dr. Anne Child guides us systematically through these non-cardiac manifestations.

With thoughtful, up-to-the-minute data, Drs. Azizzadeh and Kouchoukos and colleagues guide us through a PRO and CON debate on application of endovascular techniques in patients with vessels rendered fragile and precarious by Marfan's disease and other tissue disorders. A surprisingly balanced and practical approach emerges from this debate-in which open surgery is preferred except in special circumstances—-such as acute rupture, patch aneurysms, and open placed grafts for landing an endovascular prosthesis.

Dr. Schafers and colleagues show us that valve-sparing (either via remodeling or reimplantation) is feasible and durable even in Marfan patients.

Our group has analyzed the natural behavior of the aortic root in Marfan syndrome (MFS) patients, finding rapid growth (compared to the general thoracic aneurysm population) and a hinge point for adverse events starting at $5.5 \mathrm{~cm}$ maximal root diameter-thus supporting pre-emptive aortic root replacement well before this hinge point.

Another chapter from our group walks the reader through the open two-stage elephant trunk procedure that can be so effective vis-à-vis the progressive distally directed dilatation that occurs over time in the MFS patient.

Dr. Isselbacher and colleagues from International Registry of Acute Aortic Dissection (IRAD) provide numerous key observations regarding acute aortic dissection in the MFS patient.

Drs. Elkayam and Goland teach us key concepts regarding dangers and optimal management of pregnancy in the MFS patient.

Drs. Coselli, Weldon, Preventza, de la Cruz, and LeMaire deftly guide us through the difficult decision-making process regarding choice of valve-sparing or composite graft replacement surgery for the (often young) MFS patient, with two illustrative cases.

Drs. Estrera, Safi, and colleagues walk us through their preferred techniques for thoracoabdominal aortic replacement in MFS patients.

We are confident that—regardless of specialty or experience—each reader will find some abundant information that for him or her meets the criterion of "Tell me something I don't know".

We are grateful to Editor-in-Chief Tristan Yan for inviting us to serve as Guest Editors for this special issue on Marfan's disease.

\section{Acknowledgements}

None.

John A. Elefteriades ${ }^{1}$, MD

(Email: john.elefteriades@yale.edu)

Bulat A. Ziganshin ${ }^{1,2}, \mathrm{MD}, \mathrm{PhD}$

${ }^{1}$ Aortic Institute at Yale-New Haven Hospital, Yale University School of Medicine, New Haven, CT, USA;

${ }^{2}$ Department of Surgical Diseases \#2, Kazan State Medical University, Kazan, Russia.

(Email: bulat.ziganshin@yale.edu)

doi: $10.21037 /$ acs.2017.11.08

Conflicts of Interest: The authors have no conflicts of interest to declare.

View this article at: http://dx.doi.org/10.21037/acs.2017.11.08 